\title{
RPA and Artificial Intelligence in Budget Management Based on Multiperspective Recognition Based on Network Communication Integration
}

\author{
Haiying Luo ${ }^{1}$ and Haichang Luo $\mathbb{D}^{2}$ \\ ${ }^{1}$ College of Accounting, Zhanjiang Science and Technology College, Zhanjiang, 524094 Guangdong, China \\ ${ }^{2}$ College of Intelligent Manufacturing, Zhanjiang Science and Technology College, Zhanjiang, 524094 Guangdong, China
}

Correspondence should be addressed to Haichang Luo; luohaiying@st.btbu.edu.cn

Received 4 August 2021; Revised 25 September 2021; Accepted 30 September 2021; Published 3 November 2021

Academic Editor: Zhihan Lv

Copyright (c) 2021 Haiying Luo and Haichang Luo. This is an open access article distributed under the Creative Commons Attribution License, which permits unrestricted use, distribution, and reproduction in any medium, provided the original work is properly cited.

\begin{abstract}
Nowadays, RPA robots are increasingly used in daily office tasks such as finance and human resources. They play an increasingly important role in realizing office automation, which can improve work efficiency and reduce labor costs. In order to improve the efficiency of budget management and save human resources, this paper conducts related research based on the multiview recognition technology of network communication integration, combined with RPA in artificial intelligence technology. In the method part, this article introduces the mode of network communication integration and the principles that should be followed, as well as the related processes of RPA. In the algorithm, this paper introduces an integrated algorithm based on ELM. In the experimental part, this article predicts the performance of each model, compares identification functions with different signal-to-voice signals, and compares timing functions on different signal-to-voice signals, periodic transmission mode indicators, recognition rates of different kernel functions, and comparison of average recognition rates and multiview recognition rate comprehensive analysis of these multiple aspects. Under the same conditions, the recognition rate of some angles is lower than other angles; 0 degrees, 18 degrees, 126 degrees, and 180 degrees are slightly lower than other angles, which will affect the average recognition rate of the entire recognition. But for multiview gait features, considering the influence of each angle on the recognition rate, the characteristics of each angle are merged together, so that the recognition rate is significantly higher than the average recognition rate of 11 angles. It can be seen that multiview recognition based on network communication integration does have obvious effects on RPA and artificial intelligence in budget management and can improve the efficiency of budget management. The multiperspective recognition technology designed in this study can realize modernization and digitization in budget management.
\end{abstract}

\section{Introduction}

Now, the world economy has entered the era of knowledge economy, and budget has gradually developed into comprehensive budget management with the continuous evolution of enterprise needs. In order to gain a foothold in the increasingly fierce competitive environment and achieve good development, the company started to give more importance to corporate governance. As a modern management method, comprehensive budget management is being used by more and more companies, and more and more companies have benefited. Compared with western developed countries, my country's comprehensive budget management started late. As some central enterprises and state-owned enterprises have achieved good economic benefits through comprehensive budget management, comprehensive budget management is being accepted by more and more enterprises. Network communication integration is to integrate various separated equipment, functions, and information into interrelated, unified, and coordinated systems through 
a structured integrated distribution line system and computer network technology, so as to fully share resources.

Artificial neural networks, support vector machines, and other artificial intelligence technologies (AI) overcome the limitations of traditional models and are driven by data to fully present the nonlinear dynamic system of time series data. The universal realization of artificial intelligence technology in the field of time series analysis has proved its good performance and universal performance in the research of time series analysis problems. A large number of theoretical and empirical studies have also fully proved the authenticity and effectiveness of integrated thinking. However, the research on ensemble forecasting models is mainly focused on linear ensemble technology, and the improvement of forecasting performance is very limited.

Based on the multiperspective recognition of network communication integration, many scholars at home and abroad have conducted related research on the operation of RPA and artificial intelligence in budget management. Ivanov et al. demonstrate the impact of shared knowledge of the environment in the navigation problem of the robot group by realizing data transmission within the group and provide examples of data acquisition, distance-based resolution, and speed control methods. The scholars proposed a dynamic network formation method based on the leader replacement system. The results of the study show that sharing common information among robots in a group can significantly reduce the length of individual trajectories. The scholars have studied the related content of robots but lack comparison with other methods [1]. Murtaza et al. propose a new multiview human action recognition method using the low-dimensional motion information of the action. Before feature extraction, a preprocessing step is performed to remove noise from the contour, which is caused by imperfect but true segmentation. The results of multiview experiments using this data set provide $95.4 \%$ high accuracy using leave-one-sequence cross-validation technology. The scholars have conducted research on action recognition, but there is no clear improvement method [2]. Willcocks et al. presented a series of dilemmas faced by senior managers, thinking about the potential applications of robotic process automation in human resource functions and global business service operations. The teaching case needs to discuss the business case of RPA and cognitive automation; the case will tell us everything of the survey, how to effectively implement it in the HR and GBS environment, and whether to use RPA tactically or strategically. The authors studied the latest technology of RPA but did not make a prospect for its application field [3]. Hwang et al. introduced robotic process automation into simple repetitive task automation and started its research and development in various fields and proposed a middleware system called MIORPA to control open source RPA robots. The proposed middleware system provides a job scheduling algorithm for assigning tasks to multiple RPA robots in multiple middleware environments. The scholars also studied RPA technology but did not design specific experiments for demonstration [4]. Hassabis et al. believe that a better understanding of biological brains can play a vital role in building intelligent machines and investigate the historical interaction among $\mathrm{AI}$ and other methods inspired by neural computing research in humans and other animals, current progress. Finally, the scholars highlighted common themes that may be crucial to advancing future research in these two fields. The scholars discussed the related content of artificial intelligence but did not combine data to improve credibility [5]. Thrall et al. proposed that AI surveillance programs can help radiologists prioritize work lists by identifying suspicious or positive cases for early review. AI programs can be used to extract "radiological" information from images that cannot be recognized by visual inspection, which can add diagnostic and prognostic value from the collection of image data. The scholars' research on AI-related procedures needs to be combined with other technologies to demonstrate [6]. Piszczek believes that changes in the environment, widespread criticism, and negative views on the operation of local selfgovernment governments, coupled with a crisis of confidence in their representatives, forced management institutions to take certain actions aimed at increasing efficiency. The aim of this scientific work was to introduce the taskbased budget and budget development rules currently being carried out in Poland as a modern method of budget management. The scholar lacks a review of the mechanism of budget management [7].

This paper discusses the research methods of RPA and artificial intelligence in budget management based on the multiview recognition of network communication integration. In the method part, this article introduces the mode of network communication integration and the principles that should be followed, as well as the related processes of RPA. In fact, in this case, the performance of this model is determined by the performance, the performance of the signal-to-sound system; the signal-to-noise system is determined when the performance is based on the ELMint algorithm. In the experimental part, this article predicts the performance of each model, comparison of recognition performance under different signal-to-noise ratios, comparison of time performance under different signal-to-noise ratios, periodic transmission mode indicators, recognition rates of different kernel functions, and comparison of average recognition rates and multiview recognition rate comprehensive analysis of these multiple aspects. The novelty of this article is based on the use of the latest RPA in artificial intelligence, that is, robotic process automation technology, combined with multiview recognition in network communication integration, to improve the efficiency of budget management in the enterprise.

\section{RPA and Artificial Intelligence in Budget Management Based on Multiperspective Recognition of Network Communication Integration Methods}

2.1. Network Communication Integration. Network communication system refers to a low-cost communication system between platforms that exchange information in multiple formats such as text, image, audio, and video on the basis 
of the Internet platform and traditional communication platform and realize technical exchanges through multiple platforms and multiple terminals, and built-in highperformance communication system $[8,9]$. In practical applications, a multiclassifier ensemble can significantly improve the generalization ability of the entire system, although a multiclassifier ensemble is not perfect in theory. Designing a good integrated system is an arduous task, which is generally divided into two steps. First, design the method of base classifier generation; second, design the method of base classifier combination. Experiments have proved that in order to obtain a more ideal classification effect in an integrated system, the base classifier in the integration process generally needs to meet two characteristics: the first is diversity, and the second is accuracy [10]. If the base classifiers in the integrated system are indistinguishable, then the integration is meaningless. Network communication is to connect all isolated devices through the network and realize the communication between people, people and computers, and computers and computers through information exchange. The basic telecommunication network includes various transmission networks, such as optical cable, microwave communication, satellite communication, and access network; the telecommunication support network includes various networks or systems supporting the telecommunication network.

Communication service network includes various communication network service nodes and networks composed of service nodes, such as telephone switching network, intelligent network, data network, and multimedia network; basic telecommunication network includes various transmission networks, such as optical cable communication, mobile communication, microwave communication, satellite communication, and access network; telecommunication support network includes various networks or systems supporting telecommunication network, such as network management system, signaling network, synchronization network, billing system, call system, call center, and data center. The following principles should be followed when designing an integrated communication system: (1) ability to collect data and control functions in the field, upload data via a common interface, and understand remote control; (2) it can be through a common interface: interface, remote or local integration of on-site data and remote control, and remote upload of data integration results; (3) the communication system can be configured flexibly, multiple controllers can be integrated at the same time, and multiple servers can be implemented; (4) data can be transmitted on the local area network and the Internet; (5) system upgrades and transformations can be easily carried out, compatible with common physical networks, reduce physical network transformations, or achieve direct access; (6) can dynamically add or delete the whole online: the communication devices and servers in the system do not cause the system to stop; (7) it can be compatible with other devices and expand the system $[11,12]$. Network application integration business and industry information application integration are divided into three parts: network communication integration includes network (WAN/LAN/VPN) equipment, computer room wiring and renovation, and other integrated services; network application integration includes integrated services such as call center and network security application; industry application integration includes financial system, human resource system, warehouse management system, financial management system, online examination and evaluation system, project management system, office OA, e-commerce, industry application, and other IT system integration services. The key of system integration is to solve the interconnection and interoperability between systems. It is a multivendor, multiprotocol, and application-oriented architecture. This needs to solve all integration-oriented problems related to subsystems, building environment, construction cooperation, and organization management and staffing, such as interfaces, protocols, system platforms, and application software between various equipment and subsystems.

2.2. Multiview Recognition. Gait recognition refers to identity authentication or recognition through people's walking posture or footprints. As a kind of biometric recognition, it has the advantages of long-distance, noncontact, and difficult to imitate. The change of viewing angle or walking direction makes the extracted human contour greatly different, which is one of the main factors affecting the performance of the gait recognition system. Visual information is one of the most important sources of information for understanding the world and receiving and understanding external feedback. A large number of data studies have shown that about $75 \%$ of the information received by the brain comes from human vision, and then, the brain processes the received visual information. With the continuous progress of modern computer technology and the popularization of smart video equipment, video image information in daily life is the most important form of visual information [13]. Analyzing and recognizing human behavior in the field of computer vision has always been an extremely difficult subject. The research methods of human behavior recognition also include the joint processing of digital image information, intelligent analysis, pattern recognition, and artificial intelligence. Human behavior recognition refers to the process of using computers to process and analyze human behavior and then retrospectively identify and classify on this basis. Therefore, the first step is usually to use a suitable feature extraction method to transfer human movement information to a video or image; the second step is to use a suitable image processing method to represent this feature information; create the best for learning and learning classification model. Classification realizes the characteristics of human behavior and behavior. Human action recognition is often used in fields such as intelligent human-computer interaction, intelligent monitoring, motion and motion analysis, and video-based retrieval [14]. The method based on metric learning is also used in multiview gait recognition. The view invariant features are obtained by projecting to the subspace, and the recognition accuracy is relatively high.

Although traditional human motion recognition algorithms have achieved many results, they still have shortcomings that are affected by factors such as environment and light. In terms of smart tracking, lifestyle recognition has a very broad evolution. In the surveillance scene, the person 
being monitored often does not know that their gait is closed, and it is impossible to move in the direction we expect. Walking is initially a process of change. In the process of walking, the angle of view will change and rotate, so the angle of view is a difficult factor to control. Initially, multicore learning was proposed to control the basic matrix search and achieve optimization, but then, more and more applications are implemented in multiview data $[15,16]$. Since multicore learning uses different kernel matrices, each kernel matrix can correspond to one projection data, and the final synthetic kernel (which can be linear or nonlinear) can effectively improve performance. Subspace learning is aimed at learning from multiple angles to obtain a common latent space. The size of the investigated hidden space was smaller than all spatial dimensions in the multiprojection data. Therefore, studying spatial planning is a very effective method to overcome the curse of dimensions. Given a subdomain, the subsequent sorting and grouping tasks will be very simple. In order to overcome the challenges of viewing angle, occlusion, and the efficiency of recognizing single-view targets, multiview target recognition has attracted more and more attention. Generally speaking, the foreground of the samples in the training set is sparse and obvious. However, in practical applications, the sample perspective is not limited to the different perspectives defined by the training set. The problem is always different to solve, and it is very difficult. The simplest method is to use multiple models of a single target at the same time and finally rely on certain arbitration rules to determine the output result. However, adjusting the model parameters to adapt the single-view target model to a wider range of perspective changes usually causes the model to be cautious, leading to more false detections and recognition alarms [17]. Figure 1 shows the image effect of multiview recognition.

2.3. RPA. Automated process robots are also called RPAs and use automated process management software built into computers. This type of robot implements predesigned script flow logic, runs commercial software, and automatically performs office tasks on a computer $[18,19]$. RPA takes the robot as the virtual labor force, interacts with the existing user system, and completes the expected tasks according to the preset program. From the current technical practice, the existing RPA is only applicable to processes with high repeatability, logical determination, and relatively low stability requirements. RPA promotes the development of economic intelligence. The audit of financial statements and account books, accounting books, and electronic forms is an important link in preparing financial reports and effectively ensuring the quality of accounting information and has gradually become one of the important links in the quality of accounting information. The introduction of RPA will provide new impetus to optimize the account control process based on the general financial service model. As a robot software automation technology based on the concept of artificial intelligence, RPA is mainly based on computer scripting language and user interface implementation, and it automates processes involving multiple manual operations by repeating routine tasks. The characteristics of this technology are as follows: first, the RPA operation rules are clear,

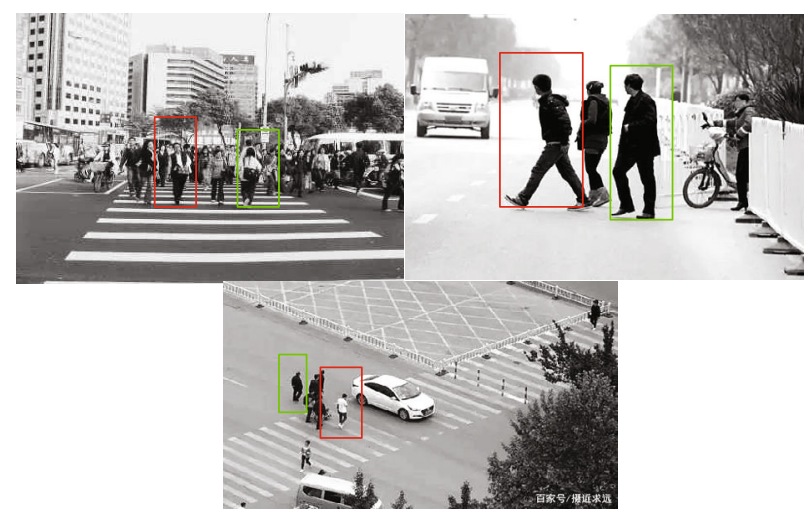

FIgURE 1: Schematic diagram of multiview recognition.

the digital activity guidelines and related process inputs are specific, and the rules are rigorous (good operation quality); the second is that RPA can simulate manual activities, interact with users, and manually integrate a large number of daily key functions. Run repeatedly. Third, RPA can provide powerful management and control capabilities. All steps of the process can be analyzed in real time (offline) and will not significantly affect the company's initial IT infrastructure and financial processes [20]. Shown in Figure 2 is the mode of robot process automation.

Process automation can enhance work roles and responsibilities and eliminate numbing responsibilities, which means that the team can improve job satisfaction, improve employee loyalty, and make them feel valuable, so as to reduce employee turnover. In the RPA process, the process programmer enters detailed instructions into the machine and issues it to the machine controller. Inspectors assign tasks to machines and monitor their activities. The machines perform tasks and interact with a large number of commercial applications. After the work is completed, business users will look at the project to identify anomalies and difficult situations. RPA resides on the existing applications of the organization. It can be used to automate routine tasks with repetitive and heavy workloads without interruption and without significant impact on the organization's traditional IT infrastructure. It can be used in any system without affecting the normal operation of each system. And it is available 24 hours a day, with powerful management, control, and supervision capabilities [21, 22]. And the process can be tracked in detail and in real time, which can make up for the inefficiency caused by the merged department. There are many repetitive and consistent links in the delayed verification process and in the audit process. Embedding RPA technology can greatly improve the efficiency of the entire audit process. At present, the application of RPA technology has become more and more mature. The application of this technology allows employees in enterprises to configure calculator software or "robot" to capture existing applications, process transactions, manipulate data, drive reaction and interaction, and communicate with other information systems. Any large amount of repetitive work performed by large-scale manpower can now be performed by the RPA software robot, saving manpower, money, and time. 


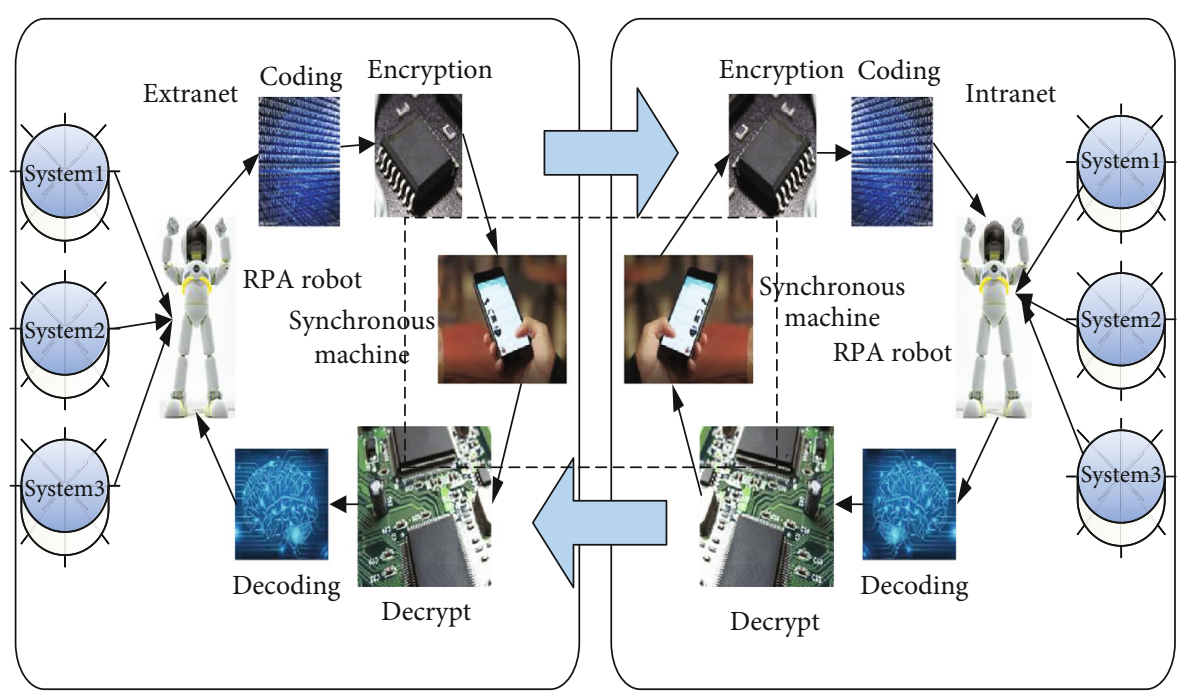

FIGURE 2: Robotic process automation.

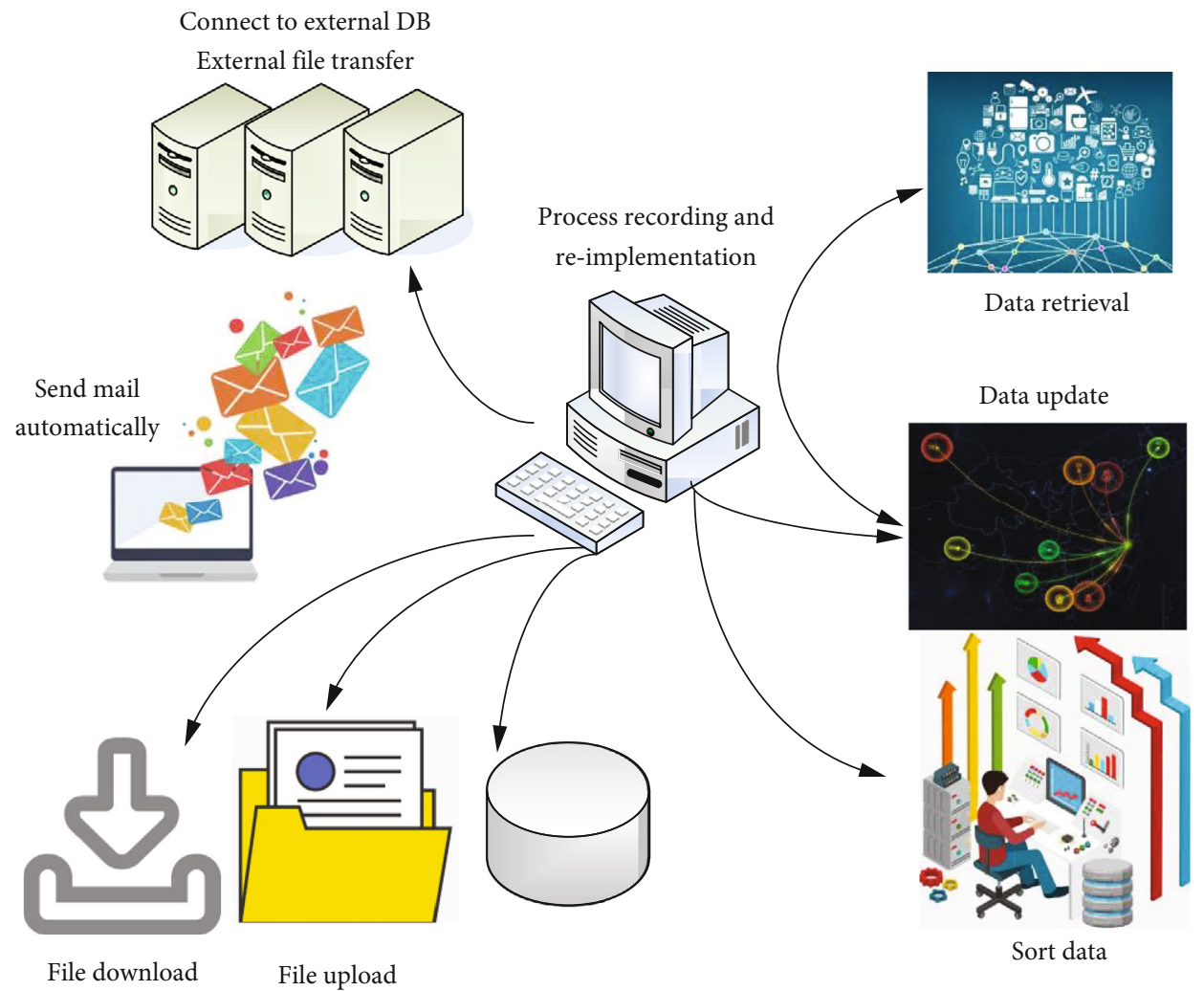

FIGURE 3: Schematic diagram of the main work of the process robot.

Shown in Figure 3 is the work content of the robot during processing.

2.4. Artificial Intelligence. With the advent of the Internet big data era and the rapid development of computer hardware technology, artificial intelligence (AI) has become an active research subject area with many practical applications and has made breakthroughs in technology and is currently developing vigorously. In particular, artificial intelligence is widely used in many fields such as face and object image rec- ognition, voice data, stock fund trend prediction, medical image data diagnosis, traffic image object recognition, and many other fields [23].

Intelligent simulation is an important task of artificial intelligence. The way artificial intelligence is realized is similar to the thinking process of the human brain. People obtain information about external conditions through the five senses, namely, vision, hearing, smell, taste, touch, etc., and form meaningful decision-making elements through the further processing of the brain. Then, through analysis 


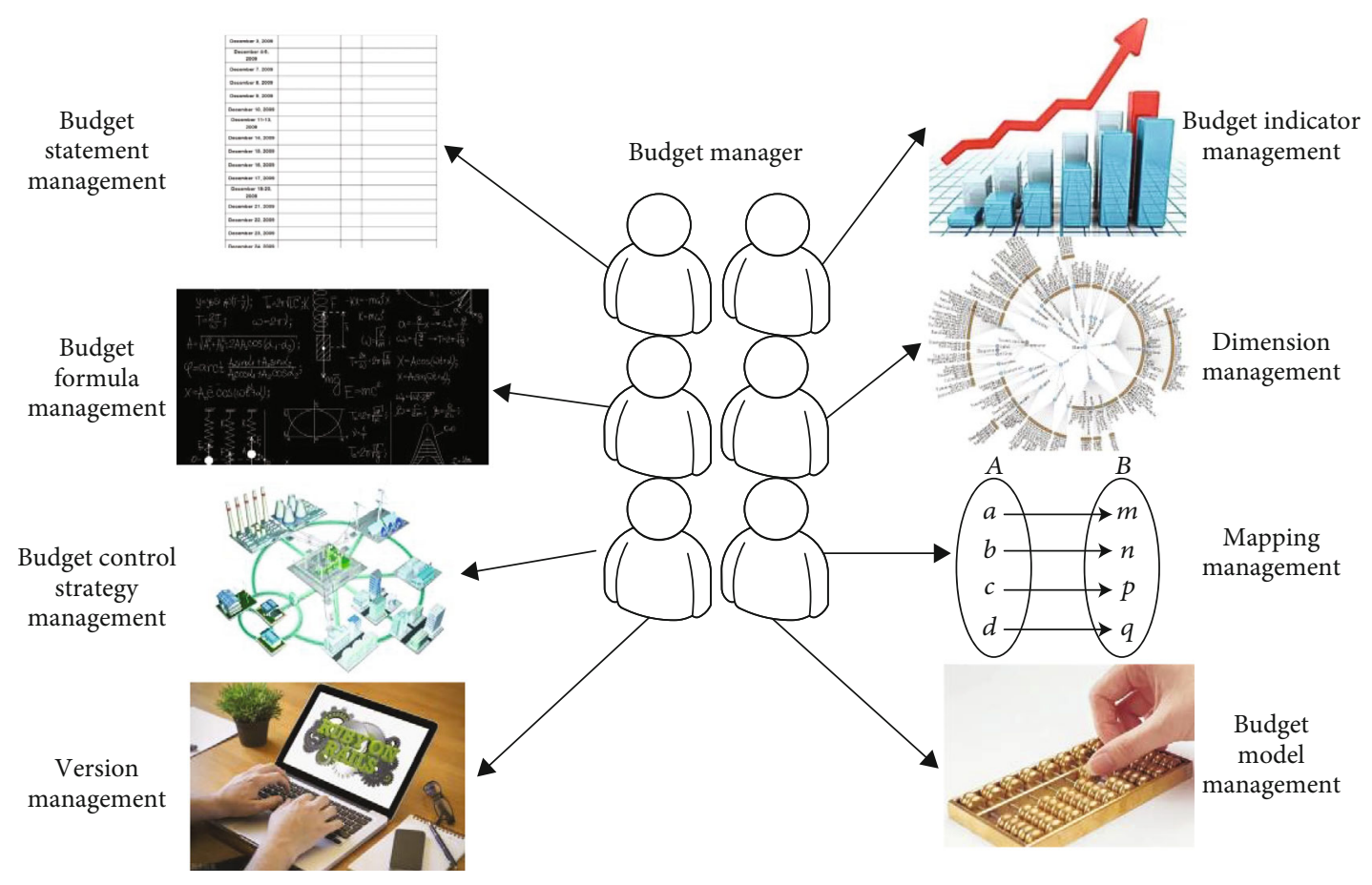

FIGURE 4: Budget reflects management use case diagram.

and reasoning and other processes, further decisions are made. The related technologies mainly include the recognition of the retina and iris, the recognition of fingerprints and palmprints, face recognition, and automatic proof of theorems, game and logical reasoning, information induction and processing, and so on. In addition to the application of artificial intelligence in sensory simulation, a more important application is to simulate the thinking and analysis process of the human brain, that is, the application of game and logical reasoning, the induction and processing of information, etc. $[24,25]$.

The most prominent neighborhood in the development of artificial intelligence technology is the research on image target recognition [26]. The accurate realization of target recognition mainly depends on the continuous improvement of the neural network structure by convolutional neural network researchers. This technology can effectively use the Internet's big data foundation and implement parallel distributed image processing training on computer hardware. The limitation of the traditional neural network method for image data processing is that it generally adopts the method of using the CPU to calculate on the computer hardware $[27,28]$.

2.5. Budget Management. Under the leadership of the general manager, the budget department is mainly responsible for organizing project bidding and preparing project budget. From the perspective of the original meaning of budget management, it is to help managers achieve their goals effectively, because at this level, budget management has important behavioral meanings. The most typical behavior in budget management is budget slack [29]. There are relatively many studies on budget slack in western scholars, many of which are carried out from the aspects of human nature, budget incentives, appraisal systems, and budget technology methods. The comprehensive budget management system includes four aspects of budget preparation, execution, supervision, and evaluation. The source and foundation of the system is budget preparation. And it will be better to take actions just towards the public and society $[30,31]$. It can be said that budgeting as the basis is of great significance in the budget management of administrative institutions and should not be underestimated. However, judging from the audit results of all levels of government by the audit department, there are still some deep-seated problems in the budget preparation work of each unit, such as incomplete budget preparation content, unreasonable budget preparation methods, and low performance in the use of fiscal funds. The supervision mechanism is not perfect, etc. Through the analysis, the main reasons are that the selection of budgeting methods is not scientific enough, the establishment of budget indicators is not specific enough, and the unit lacks a set of scientific and effective budgeting system [32, 33].

The budget system management business mainly includes eight subfunctions: budget index management, dimension management, mapping management, budget model management, budget form management, budget formula management, budget control strategy management, and version management. They jointly complete the formulation of budget model control strategies. Figure 4 shows the flow chart of the use of budget management [34]. From the perspective of budget managers, Figure 4 involves eight aspects: budget report management, formula management, and control strategy management.

2.6. Integration Algorithm Based on ELM. When initializing the weights, the "leave one method" is used instead of the "unified initialization method," and the actual output and the leave one error are used to calculate the leave one 
TABLE 1: Model performance of each time series forecasting model.

\begin{tabular}{lcccc}
\hline Evaluation index & NMSE & Rank & $S$ & Rank \\
\hline ARMA-TMKR & 3.2178 & 6 & $45 \%$ & 5 \\
D-KPNN & 1.0234 & 2 & $46 \%$ & 4 \\
LMD-SVM & 1.5276 & 5 & $52 \%$ & 3 \\
Simple average ensemble forecast & 1.2483 & 4 & $54 \%$ & 2 \\
KPNN & 1.1347 & 3 & $52 \%$ & 3 \\
SVM & 0.7472 & 1 & $64 \%$ & 1 \\
\hline
\end{tabular}

method output, and the drop one is calculated by the last error which is also dropped [35]. The leave-one-out error calculation method is

$$
\chi_{x,-x}=n_{x}-m_{x} a_{-x}=n_{x}-\widehat{n}_{x,-x} .
$$

Among them, $x$ is a variable. In the training phase, the training data can be trained individually or in groups. In this model, the model parameters are determined by continuous data. Suppose a set of data $M$ :

$$
M_{1}=\left\{\left(m_{x}, s_{x}\right)\right\}_{x=J_{0}+1}^{J_{0}+J_{1}} .
$$

When we are going to make the result of formula (3), minimizing the variance becomes

$$
s_{\min }=2\left\|\left[\begin{array}{l}
L_{0} \\
L_{1}
\end{array}\right] \alpha-\left[\begin{array}{l}
K_{0} \\
K_{1}
\end{array}\right]\right\| .
$$

Among them,

$$
\begin{aligned}
L_{1} & =\left[\begin{array}{ccc}
F\left(c_{1}, a_{1}, m_{J_{0}+1}\right) & \cdots & F\left(c_{\hat{J}}, a_{\hat{J}}, m_{J_{0}+1}\right) \\
\vdots & \cdots & \vdots \\
F\left(c_{1}, a_{1}, m_{J_{0}+J_{1}}\right) & \cdots & F\left(c_{\hat{J}}, a_{\hat{J}}, m_{J_{0}+J_{1}}\right)
\end{array}\right], \\
S & =\left[\begin{array}{c}
s_{J_{0}+1}^{S} \\
\vdots \\
s_{J_{0}+J_{1}}^{S}
\end{array}\right]_{J_{1} \times i} .
\end{aligned}
$$

Considering the sum of two sets of data $M_{1}$ and $M_{2}$, and we can not see the meaning of the index of $D$, the output weight $\alpha$ becomes

$$
\alpha^{(1)}=D_{1}^{-1}\left[\begin{array}{l}
L_{0} \\
L_{1}
\end{array}\right]^{S}\left[\begin{array}{l}
S_{0} \\
S_{1}
\end{array}\right] .
$$

Here,

$$
D_{1}=\left[\begin{array}{c}
L_{0} \\
L_{1}
\end{array}\right]^{S}\left[\begin{array}{c}
L_{0} \\
L_{1}
\end{array}\right]
$$

In sequential learning, it can be expressed as a function of and not a function of the data set, written as follows.

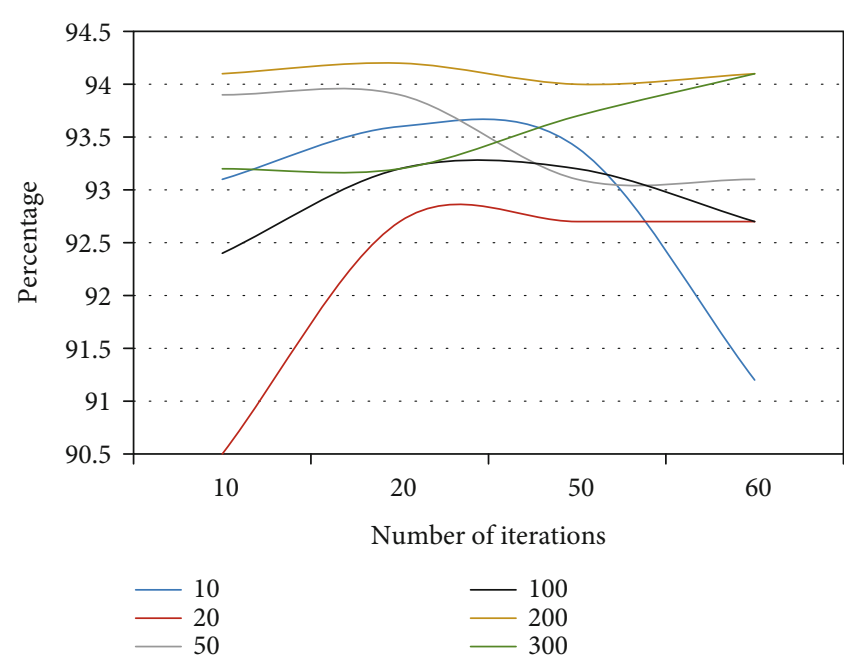

FIgURE 5: Radiation source recognition rate of a double-layer common automatic encoder.

In sequential learning, $\alpha^{(1)}$ can be expressed as a function of $\alpha^{(0)}, D_{1}$ and $L_{1}$ are not functions of data set $M_{1}$, and $D_{1}$ is written as

$$
D_{1}=\left[L_{0}{ }^{S} L_{1}^{S}\right]\left[\begin{array}{l}
L_{0} \\
L_{1}
\end{array}\right]=D_{0}+L_{1}{ }^{S} L_{1} .
$$

At the same time,

$\left[\begin{array}{l}L_{0} \\ L_{1}\end{array}\right]^{S}\left[\begin{array}{l}L_{0} \\ L_{1}\end{array}\right]=L_{0}^{S} S_{0}+{ }_{1}=D_{1} \alpha^{(0)}-L_{1}^{S} L_{1} \alpha^{(0)}+L_{1}^{S} S$.

Combine the above formula to get

$$
\alpha^{(0)}=D_{1}^{-1}\left[\begin{array}{l}
L_{0} \\
L_{1}
\end{array}\right]^{S}\left[\begin{array}{l}
L_{0} \\
L_{1}
\end{array}\right]=\alpha^{(0)}+D_{1}^{-1} L_{1} S\left(S_{1}-L_{1} \alpha^{(0)}\right) \text {. }
$$

Here, define $D_{1}$ as

$$
D_{1}=D_{0}+L_{1}^{S} L_{1}
$$

When $d+1$ sets of data are trained, there are

$$
D_{d+1}^{-1}=\left(D_{d}+H_{d+1}^{S} H_{d+1}\right)^{-1} .
$$

Suppose that $Q_{d+1}=D_{d+1}^{-1}$, but we found that the number is not enough for us to use, and then, the updated formula of $\alpha^{(d+1)}$ is

$$
\begin{aligned}
Q_{d+1} & =Q_{d}-Q_{d} L_{d+1}^{S}\left(T+L_{d+1} Q_{d}^{-1} L_{d+1}^{S}\right)^{-1} L_{d+1} Q_{d}, \\
\alpha^{(d+1)} & =\alpha^{(d)}+Q_{d+1} L_{d+1}^{S}\left(S_{d+1}-L_{d+1} \alpha^{(d)}\right) .
\end{aligned}
$$




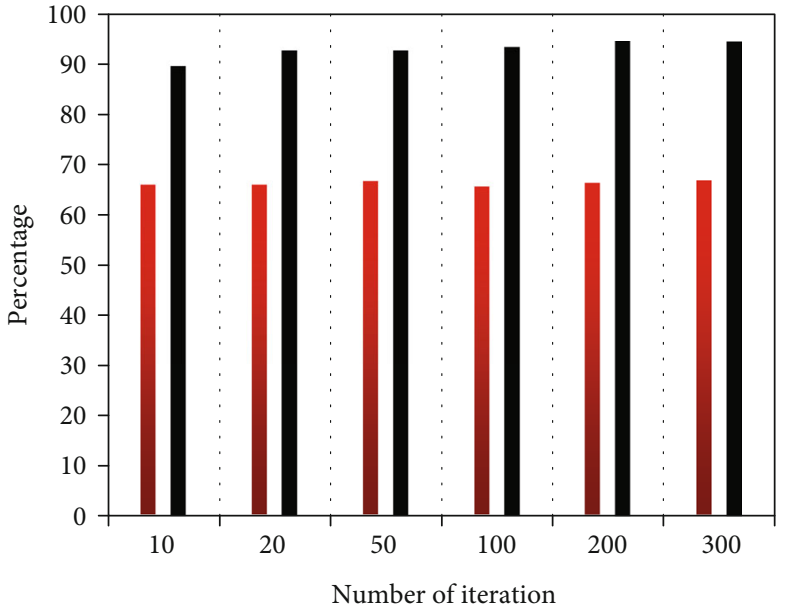

$\square \mathrm{CNN}$

(a) The signal-to-noise ratio is $-10 \mathrm{~dB}$

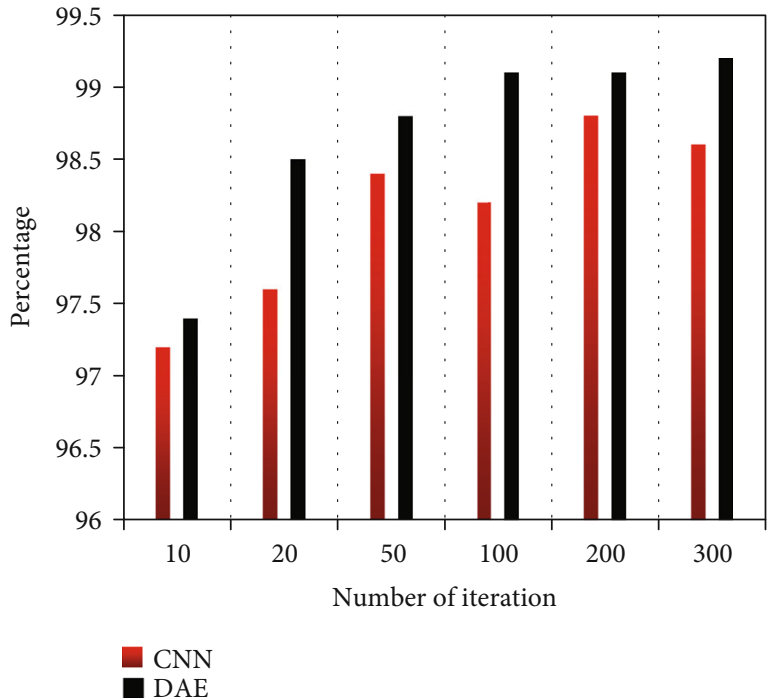

(b) The signal-to-noise ratio is $10 \mathrm{~dB}$

FIGURE 6: Radiation source recognition rate under two kinds of neural networks.

The output of neural network integration is $g^{y}\left(m_{x}\right), y$ $=1, \cdots, Q$, which can be obtained through integration:

$$
g\left(m_{x}\right)=\frac{1}{Q} \sum_{y=1}^{Q} g^{(y)}\left(m_{x}\right)
$$

Among this, $g\left(m_{x}\right)$ represents the output of the input sample $m_{x}$ on the entire system.

\section{RPA and Artificial Intelligence in Budget Management Based on Multiperspective Recognition Based on Network Communication Integration Experiments and Result}

3.1. Sources of Experimental Data. IXMAS data collection is a collection of human body movement data with multiple view views. This data set contains thirteen groups of daily actions, which were repeated three times by eleven volunteers. When this data set was recorded, it was recorded by five cameras and provided in the form of pictures. The resolution of each picture was $390 * 291$.

3.2. Predictive Performance of Each Model. From the perspective of two evaluation indicators, the standardized mean square error NMSE and the direction change statistic $S$, Table 1 shows the specific performance of series forecasting models and lists the ranking of each model on the two indicators. As can be seen from Table 1, there are three separate prediction models, namely, ARMA-TMKR model, D-KPNN model, and LMD-SVM model, and three integrated prediction models, namely, simple average integrated model, KPNN integrated prediction model, and SVM integrated prediction model. For classification, the SVM model ranks first and ARMA-TMKR ranks last. The prediction performance of the SVM integrated prediction model is the best among the six models, not only is NMSE the smallest, but $S$ is the largest.

Figure 5 shows the overall recognition rate of the duallayer autoencoder when the learning rate is 1 under the condition of $0 \mathrm{~dB}$ signal-to-noise ratio. The goal is to compare performance with automatic tiering.

3.3. Comparison of Recognition Performance under Different Signal-to-Noise Ratios. Time performance is evaluated in terms of time consumption, while recognition performance is evaluated in terms of recognition effect. Figure 6 shows the radiator recognition rate of the convolutional neural network and the noise reduction autoencoder under the conditions of $-5 \mathrm{~dB}$ and $5 \mathrm{~dB}$ signal-to-noise ratio. It can be seen from the figure that when the signal-to-noise ratio is low, the denoising autoencoder has a better recognition rate than the convolutional neural network; and the figure also shows that under the condition of a high signal-to-noise ratio, the volume of the recognition efficiency of the product neural network is also very good, even better than the noise reduction autoencoder, and of course, the noise reduction autoencoder is not bad at all. However, in a more complex electromagnetic environment, such as the incomplete acquisition of the radiation source information or the lack of information, the noise reduction automatic encoder will have more advantages [36].

3.4. Comparison of Time Performance under Different Signalto-Noise Ratios. Figures $7(\mathrm{a})$ and $7(\mathrm{~b})$ are the timeconsuming training of the two neural networks under the conditions of $-5 \mathrm{~dB}$ and $5 \mathrm{~dB}$ signal-to-noise ratio, respectively. CNN is a feedforward neural network, which usually includes data input layer, convolution calculation layer, relu activation layer, pooling layer, and full connection layer. It is a neural network in which convolution operation replaces traditional matrix multiplication operation. The comparison shows that the training of denoising autoencoders in low 


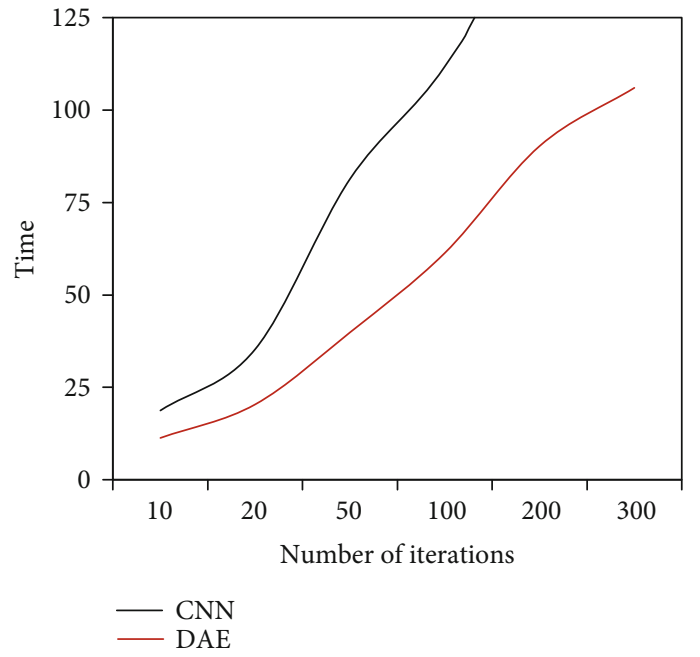

(a) The signal-to-noise ratio is $-10 \mathrm{~dB}$

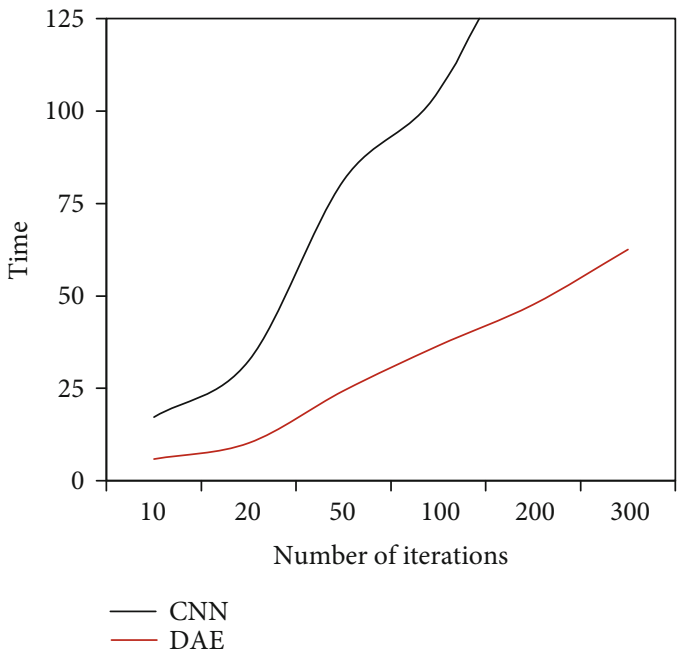

(b) The signal-to-noise ratio is $10 \mathrm{~dB}$

Figure 7: Time performance under two kinds of neural networks.

TABle 2: Periodic transmission mode index parameters under different network capacities.

\begin{tabular}{lccc}
\hline $\begin{array}{l}\text { Network } \\
\text { capacity }\end{array}$ & $\begin{array}{c}\text { Channel } \\
\text { utilization }\end{array}$ & $\begin{array}{c}\text { Packet } \\
\text { length }\end{array}$ & $\begin{array}{c}\text { Maximum sampling } \\
\text { rate of single channel }\end{array}$ \\
\hline 1 & $21.76 \%$ & 125 & 1756 \\
5 & $21.23 \%$ & 125 & 1287 \\
10 & $21.34 \%$ & 125 & 676 \\
20 & $21.61 \%$ & 125 & 354 \\
50 & $21.92 \%$ & 125 & 186 \\
100 & $21.54 \%$ & 125 & 76 \\
\hline
\end{tabular}

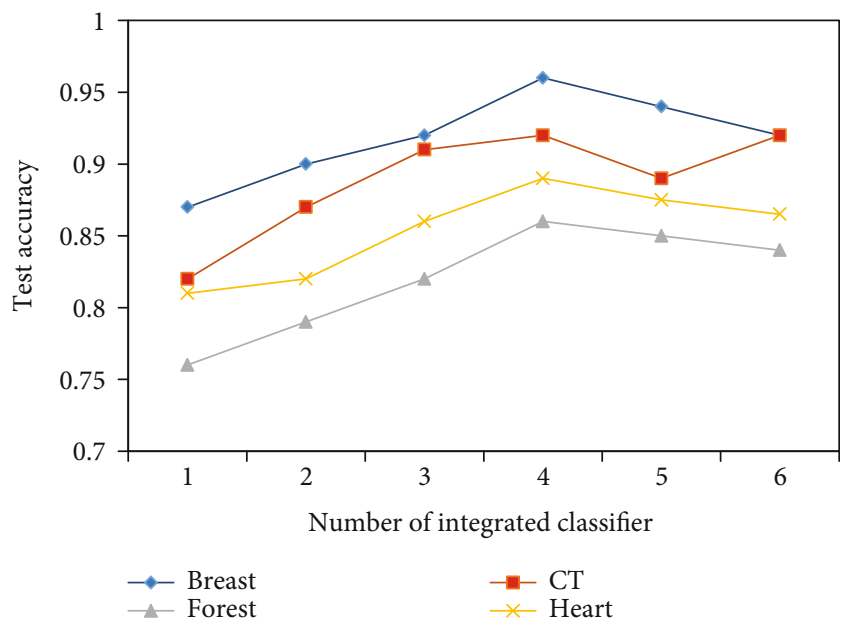

FIgURE 8: The relationship between the number of integrated classifiers and test accuracy.

signal-to-noise ratio conditions takes more time, but it is twice as efficient as convolutional neural networks. This causes that the networks go to work with a wrong direction and situation.
TABLE 3: Recognition rate of different kernel functions.

\begin{tabular}{lccccc}
\hline \multirow{2}{*}{ Kernel function } & \multirow{2}{*}{ Linear } & \multicolumn{5}{c}{ Polynomial } & \multirow{2}{*}{ Gauss } \\
& & $q=1$ & $q=2$ & $q=3$ & \\
\hline $\begin{array}{l}\text { Test sample point } \\
\begin{array}{l}\text { Correctly identify the } \\
\text { number }\end{array}\end{array}$ & 368 & 368 & 368 & 368 & 368 \\
Recognition rate & 253 & 287 & 303 & 311 & 322 \\
\hline
\end{tabular}

3.5. Periodic Transmission Mode Indicators. For example, Table 2 shows the index parameters of the periodic transmission mode under different network capacities. The maximum values of the four index parameters are when the network capacities of $1,5,10,20,50$, and 100 are extracted. In the periodic transmission mode, when the network is working at high speed, no matter how large the network capacity is, the channel utilization rate tends to be consistent, maintaining around $21 \%$.

The results clearly show the relationship between the number of integrated classifiers and the test accuracy, as shown in Figure 8. As the number of integrated classifiers increases, the accuracy of the test will increase. But it will increase to a certain extent. When the number of classifiers reaches 4 , the test accuracy will not be greatly improved. Some may be slightly reduced.

3.6. Recognition Rate of Different Kernel Functions. It can be seen from Table 3 that under the same experimental conditions, the recognition rate of the Gaussian kernel function is slightly higher than that of the other two methods. Gaussian kernel function, linear, and polynomial are all functions involved in support vector machine. Therefore, in the subsequent experiments, each group of experiments uses the Gaussian kernel function.

3.7. Comparison of Average Recognition Rate and Multiview Recognition Rate. It can be seen from Figure 9 that under the same conditions, the recognition rate of some angles is lower 


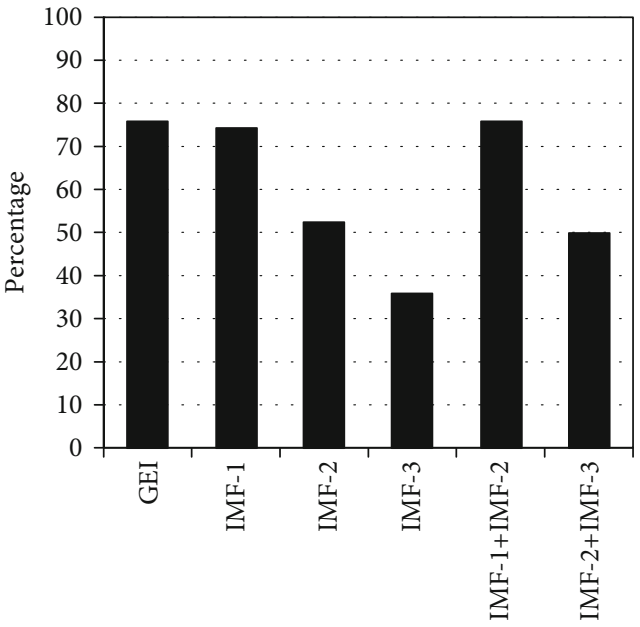

Method

Average recognition rate

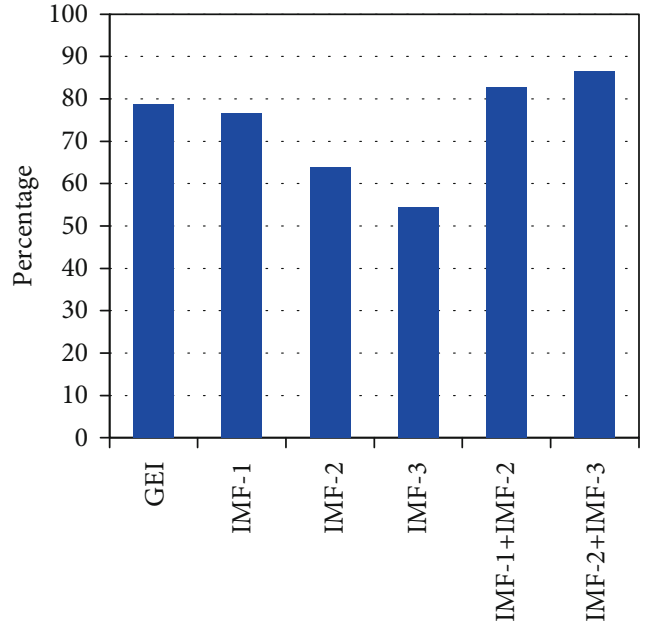

Method

Multi-view recognition rate

FIGURE 9: Comparison of average recognition rate and multiview recognition rate.

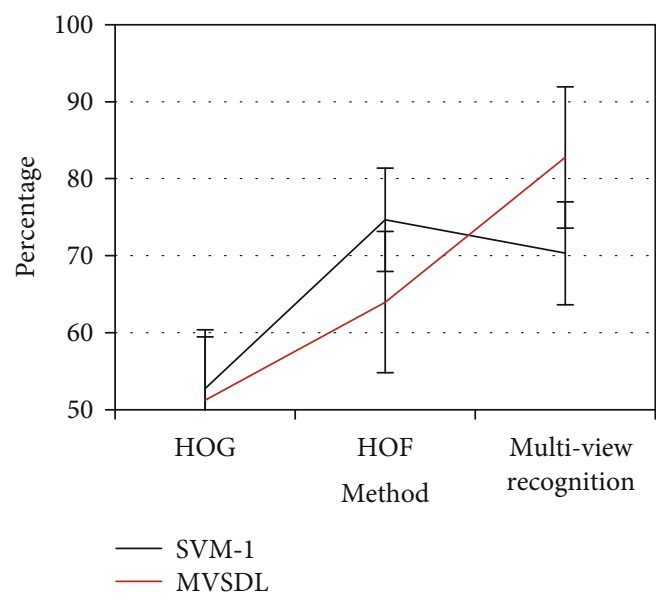

(a) $l=3$ (1 time for each action)

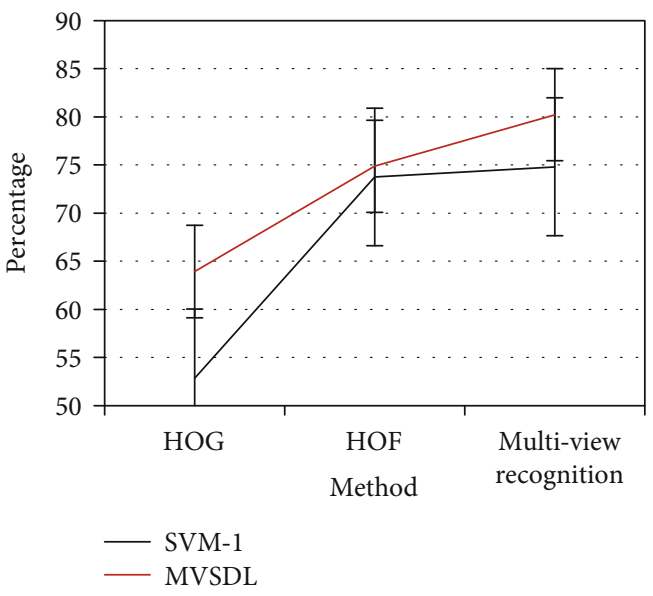

(b) $l=9$ (3 times for each action)

FIgURE 10: Comparison of recognition rates of classification methods under multiple perspectives.

TABLE 4: MSR Action3D action recognition comparison results.

\begin{tabular}{lcccccc}
\hline & 1 & 2 & 3 & 4 & 5 & 6 \\
\hline Action set 1 & 0.723 & 0.856 & 0.912 & 0.823 & 0.759 & 0.811 \\
Action set 2 & 0.764 & 0.835 & 0.518 & 0.758 & 0.776 & 0.836 \\
Action set 3 & 0.856 & 0.624 & 0.937 & 0.866 & 0.952 & 0.904 \\
Average & 0.753 & 0.776 & 0.824 & 0.813 & 0.845 & 0.873 \\
\hline
\end{tabular}

than that of other angles. 0 degrees, 18 degrees, 126 degrees, and 180 degrees are all slightly lower than other angles, which will affect the average recognition rate. But for multiview gait features, considering the influence of each angle on the recognition rate, the characteristics of each angle are merged together, so that the recognition rate is significantly higher than the average recognition rate of 11 angles.

As shown in Figure 10, the recognition rate of each classification method under multiple perspectives is compared. Action recognition under multiple perspectives refers to a motion action instance described by using action features from multiple perspectives. Usually, multiple camera devices can be used. To obtain action features from multiple perspectives, this paper uses Harris 3D detectors to obtain spatiotemporal interest points of action features in action videos in the KTH database.

Table 4 and Figure 11 show the comparison of different MSR Action3D action recognition methods. These six methods include the traditional methods and the algorithms used in this paper, of which 6 are the algorithms in this paper. The sixth algorithm is based on the average classification results under the three data sets and can achieve better classification results in different classification data.

\section{Discussion}

Compared with the multiview spatial position information fusion method that directly uses spatial affine transformation for multiview target association, this method calculates 


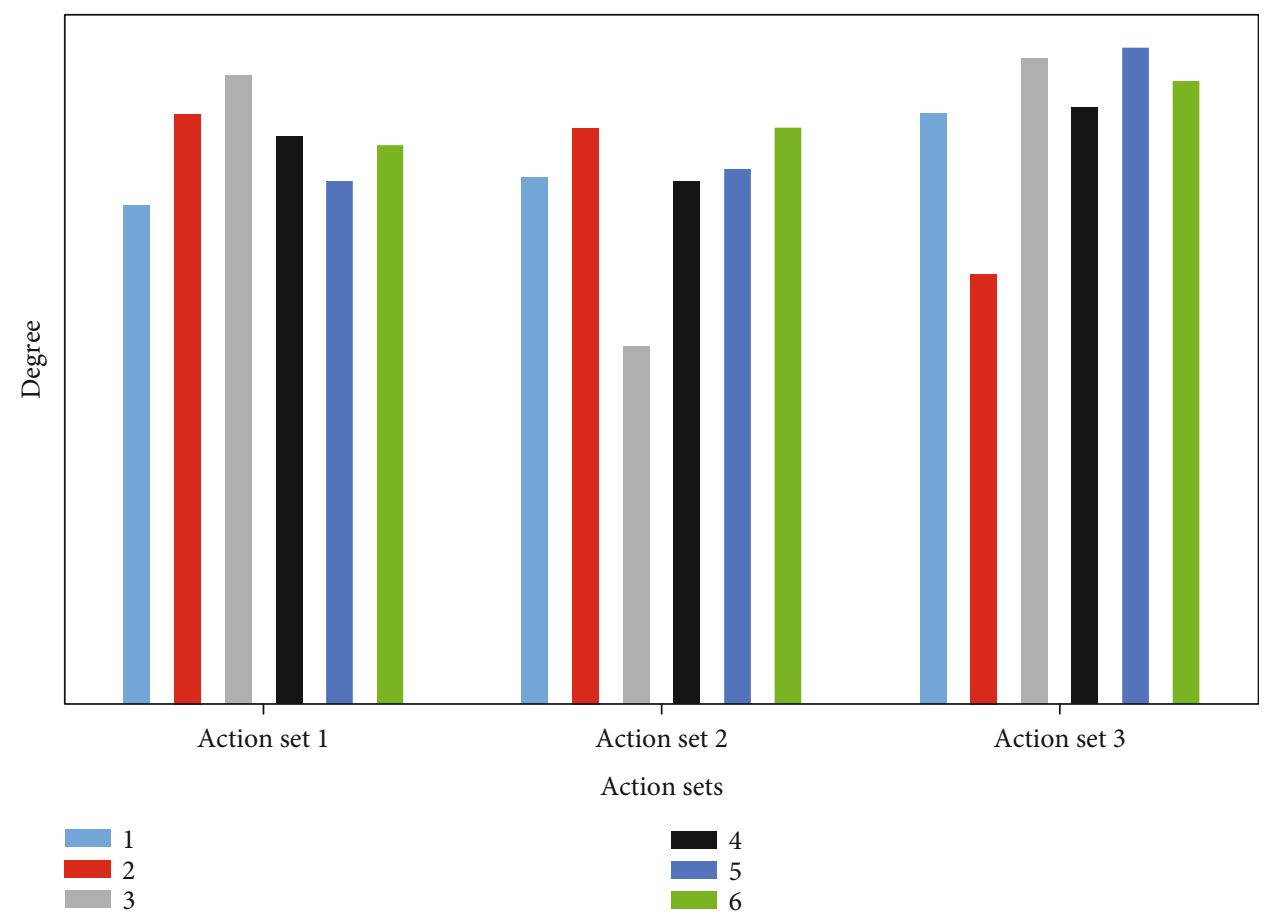

FIgURE 11: MSR Action3D action recognition comparison results.

the probability of the target appearing in different positions under each view, so as to achieve more flexible multiview association, which is beneficial to further avoid missed inspection under single viewing angle, so the recall rate can be further improved. Like the multiview spatial location information fusion method, this method cannot solve the mutual interference of occluded targets, so the target detection accuracy is low. The method proposed in this paper avoids the dependence of the first two methods on spatial location information and directly uses the visual features of the target area for multiview association, so that it knows the combination of multi-image works and imagination, so this method can further improve the recall rate; secondly, this method the multiview target matching is transformed into the maximum posterior probability problem based on the structural constraints of the human body block area, and the multiview target matching result is obtained through the optimal solution, so it can effectively solve the detection difficulty caused by the multitarget occlusion.

Using RPA budgeting can simplify manual accounting procedures, save manpower and material resources, and reduce the cost of accounting information. It provides quality assurance for accounting work and improves management efficiency. In terms of the settlement process, RPAbased payment applications can help the payment team to dynamically and real-time control the accounts payable funds, which is convenient and safe, improves the efficiency of fund payment, and reduces costs and risks. Analyze the repayment of mutual fund debt. Due to the regularity and high repetition rate of data reporting, based on this, the implementation of RPA will be able to complete the data reporting task in the feedback report. When exporting data, RPA will prepare a unique identifier for reference data and accounting data according to predefined rules. After completing the audit of the financial sharing center account, create the latest data items. The RPA completes the update data in the corresponding data elements in sequence according to the unique data element ID of the data element, thereby completing the completion of the feedback reference data.

\section{Conclusion}

If we want to better balance the relationship between efficiency and cost reduction, we can either directly replace human beings with automation or continuously optimize the structure of human resources. Automation is often our first choice, even if a robot is valuable. Automation technology continues to change, and RPA is just popular in this era, so RPA has become the focus of financial executives. Robotic process automation is a very important emerging technology in artificial intelligence theory. For this technology, two causal mechanisms are implemented: prelink and postlink. By improving RPA, decision-making requirements can be included in business management. Change the user interface and perform visual interactive simulation. Therefore, management can see how the model works and how it interacts with decision-making and management. Or research interactive employees can also use their knowledge to make judgments and try different strategies. This is very useful for controlling selection and defining business processes during the interaction with the model. Based on the multiperspective recognition of network communication integration, this paper studies the operation of RPA in artificial intelligence in budget management, predicting performance from various models, comparing recognition performance under different signal-to-noise ratios, and comparing time performance under 
different signal-to-noise ratios, and periodic transmission A certain analysis has been made on the pattern indicators, the recognition rate of different kernel functions, and the comparison of the average recognition rate and the multiview recognition rate. The detection speed of the work is much worse than other aspects; it makes different identities, such as 0 degrees, 18 degrees, 126 degrees, and 180 degrees which are slightly lower than other angles, which will affect the average recognition rate of the entire recognition. But for the multiview gait feature, the influence of each angle on the recognition rate is comprehensively considered, and the characteristics of each angle are merged together. The shortcoming of this article is that this article does not have a practical identification scheme for the design of machine process automation, that is, it does not design the input and output research of the physical model for identification. It is hoped that with the in-depth research on the network communication integration technology, these problems can be gradually improved.

\section{Data Availability}

Data sharing does not apply to this article because no data set was generated or analyzed during the current research period.

\section{Conflicts of Interest}

The authors declare that this article has no competing interests.

\section{Acknowledgments}

This work was supported by the project of "Guangdong Province Key Cultivation Discipline-Accounting” (Yuejiao Yanhan No.1, key cultivation, serial No. 11), "Private University Budget Management Optimization Research" (Academy (2019)39,40, CJ19CXQX001), the innovation and application of the Practical Teaching System of Accounting Specialty (No. 72, JY2020002, school teaching and development 2020), the VBSE Teaching Team of Accounting Comprehensive Simulation Experiment (No. 47, Zlgc2019002, school teaching and development 2020), "Accounting Comprehensive Simulation Experiment" "Course Ideological and Political" Demonstration Course (school teaching and development 27[2021]27, PPJH202126YLKC), “Accounting” Key Specialty (school teaching and development 27[2021], PPJH2021001), and Brand Promotion Project of Zhanjiang Science and Technology College: Digital Intelligence Finance Teaching Team (project No.: PPJH202106JXTD).

\section{References}

[1] M. Ivanov, O. Sergiyenko, V. Tyrsa et al., "Software advances using $\mathrm{n}$-agents wireless communication integration for optimization of surrounding recognition and robotic group dead reckoning," Programming and Computer Software, vol. 45, no. 8, pp. 557-569, 2019.

[2] F. Murtaza, M. H. Yousaf, and S. A. Velastin, "Multi-view human action recognition using $2 \mathrm{D}$ motion templates based on MHIs and their HOG description," IETuter Vision, vol. 10, no. 7, pp. 758-767, 2016.

[3] L. Willcocks, M. Lacity, and A. Craig, "Robotic process automation: strategic transformation lever for global business services?," Journal of Information Technology Teaching Cases, vol. 7, no. 1, pp. 17-28, 2017.

[4] M. H. Hwang, U. K. Na, S. Lee et al., "MIORPA: middleware system for open-source robotic process automation," Journal of Computing Science and Engineering, vol. 14, no. 1, pp. 19$25,2020$.

[5] D. Hassabis, D. Kumaran, C. Summerfield, and M. Botvinick, "Neuroscience-inspired artificial intelligence," Neuron, vol. 95, no. 2, pp. 245-258, 2017.

[6] J. H. Thrall, X. Li, Q. Li et al., "Artificial intelligence and machine learning in radiology: opportunities, challenges, pitfalls, and criteria for success," Journal of the American College of Radiology, vol. 15, no. 3, pp. 504-508, 2018.

[7] M. Piszczek, "Budget management in self-government-Polish case," Journal of WEI Business and Economics, vol. 7, no. 2, pp. 14-30, 2018.

[8] A. Medina-Santiago, "Web application development by applying the MVC and table data gateway in the annual program budget management system," International Journal of Advanced Computer Science and Applications, vol. 8, no. 2, pp. 250-255, 2017.

[9] S. Chen, S. Sun, and S. Kang, "System integration of terrestrial mobile communication and satellite communication - the trends, challenges and key technologies in B5G and 6G," China Communications, vol. 17, no. 12, pp. 156-171, 2020.

[10] Z. Dou, X. Zhong, and W. Zhang, "Radar-communication integration based on MSK-LFM spread spectrum signal," International Journal of Communications Network \& System Sciences, vol. 10, no. 8, pp. 108-117, 2017.

[11] T. Zhang, G. Han, C. Lin, N. Guizani, H. Li, and L. Shu, "Integration of communication, positioning, navigation and timing for deep-sea vehicles," IEEE Network, vol. 34, no. 2, pp. 121$127,2020$.

[12] M. A. Hannah and M. Simeone, "Exploring an ethnographybased knowledge network model for professional communication analysis of knowledge integration," IEEE Transactions on Professional Communication, vol. 61, no. 4, pp. 372-388, 2018.

[13] M. Sicilia and J. Merino, "Integración de la estrategia de comunicación en canales propios: El caso de El Corte Inglés," aDResearch: Revista Internacional de Investigación en Comunicación, vol. 21, no. 21, pp. 8-26, 2020.

[14] V. K. Tadia, "Implementation and integration of picture archiving and communication systems (PACS) at tertiary care hospitals in India," International Journal of Scientific Research, vol. 9, no. 2, pp. 64-67, 2020.

[15] Y. H. Yuan, Y. Li, J. Liu et al., "Learning multi-kernel multiview canonical correlations for image recognition," Computational Visual Media, vol. 2, no. 2, pp. 153-162, 2016.

[16] S. Jiang, W. Min, L. Liu, and Z. Luo, "Multi-scale multi-view deep feature aggregation for food recognition," IEEE Transactions on Image Processing, vol. 29, no. 1, pp. 265-276, 2020.

[17] X. Ji, Z. Ju, C. Wang, and C. Wang, "Multi-view transition HMMs based view-invariant human action recognition method," Multimedia Tools and Applications, vol. 75, no. 19, pp. 11847-11864, 2016.

[18] A. A. Liu, N. Xu, W. Z. Nie, Y. T. Su, Y. Wong, and M. Kankanhalli, "Benchmarking a multimodal and multiview 
and interactive dataset for human action recognition," IEEE Transactions on Cybernetics, vol. 47, no. 7, pp. 1781-1794, 2017.

[19] P. J. Matthias and J. Herrmann, "Einsatz von robotic process automation (RPA) im Energiehandel," Energiewirtschaftliche tagesfragen: Zeitschrift fur energiewirtschaft, recht, technik, und umwelt, vol. 68, no. 7-8, pp. 54-55, 2018.

[20] A. Asatiani and E. Penttinen, “Turning robotic process automation into commercial success - case OpusCapita," Journal of Information Technology Teaching Cases, vol. 6, no. 2, pp. 67-74, 2016.

[21] R. Li, Z. Zhao, X. Zhou et al., "Intelligent 5G: when cellular networks meet artificial intelligence," IEEE Wireless Communications, vol. 24, no. 5, pp. 175-183, 2017.

[22] S. Kulkarni, "Cloud data pipeline automation framework with integrated budget management," International Journal of Advanced Trends in Computer Science and Engineering, vol. 9, no. 4, pp. 5554-5554, 2020.

[23] V. V. Shevchuk, N. Y. Melnichuk, and A. S. Krutova, "Proactive mechanism of budget management in the state regulation of the economy," Financial and Credit Activity Problems of Theory and Practice, vol. 2, no. 29, pp. 476-486, 2019.

[24] N. Y. Melnychuk, N. O. Parfentseva, and I. O. Melnychuk, "Statistical assessment of the budget management effectiveness when drawing up, reviewing and approving the state budget," Statistics of Ukraine, vol. 86, no. 3, pp. 38-46, 2019.

[25] S. Kim, "5G network communication, caching, and computing algorithms based on the two-tier game model," ETRI Journal, vol. 40, no. 1, pp. 61-71, 2018.

[26] H. Shen, M. Zhang, H. Wang, F. Guo, and W. Susilo, “A cloudaided privacy-preserving multi-dimensional data comparison protocol," Information Sciences, vol. 545, no. 1, pp. 739-752, 2021.

[27] T. Sui, D. Marelli, X. Sun, and M. Fu, "Multi-sensor state estimation over lossy channels using coded measurements," Automatica, vol. 111, article 108561, 2020.

[28] L. Ding, L. Huang, S. Li et al., "Definition and application of variable resistance coefficient for wheeled mobile robots on deformable terrain," IEEE Transactions on Robotics, vol. 36, no. 3, pp. 894-909, 2020.

[29] Y. Yang, C. Hou, Y. Lang, T. Sakamoto, Y. He, and W. Xiang, "Omnidirectional motion classification with monostatic radar system using micro-Doppler signatures," IEEE Transactions on Geoscience and Remote Sensing, vol. 58, no. 5, pp. 35743587, 2020.

[30] Y. He, L. Dai, and H. Zhang, "Multi-branch deep residual learning for clustering and beamforming in user-centric network," IEEE Communications Letters, vol. 24, no. 10, pp. 2221-2225, 2020.

[31] X. Cheng, B. Yang, T. Olofsson, G. Liu, and H. Li, “A pilot study of online non-invasive measuring technology based on video magnification to determine skin temperature," Building and Environment, vol. 121, pp. 1-10, 2017.

[32] S. Yang, B. Deng, J. Wang et al., "Scalable digital neuromorphic architecture for large-scale biophysically meaningful neural network with multi-compartment neurons," IEEE Transactions on Neural Networks and Learning Systems, vol. 31, no. 1, pp. 148-162, 2020.

[33] B. Yang, X. Li, Y. Hou et al., "Non-invasive (non-contact) measurements of human thermal physiology signals and thermal comfort/discomfort poses -a review," Energy and Buildings, vol. 224, article 110261, 2020.

[34] X. Zheng and Z. Cai, "Privacy-preserved data sharing towards multiple parties in industrial IoTs," IEEE Journal on Selected Areas in Communications, vol. 38, no. 5, pp. 968-979, 2020.

[35] Z. Cai, Z. Xiong, H. Xu, P. Wang, W. Li, and Y. Pan, "Generative adversarial networks," ACM Computing Surveys, vol. 54, no. 6, pp. 1-38, 2021.

[36] M. Jahanbakht, W. Xiang, L. Hanzo, and M. Rahimi Azghadi, "Internet of underwater things and big marine data analytics-a comprehensive survey," IEEE Communications Surveys \& Tutorials, vol. 23, no. 2, pp. 904-956, 2021. 